\title{
BALANCE CUALITATIVO DE 50 AÑOS DE INTERVENCIÓN ARQUEOLÓGICA EN SEVILLA (1944-1998)
}

\section{A QUALITATIVE ASSESSMENT OF 50 YEARS OF RESCUE ARCHAEOLOGY IN SEVILLA (1944-1998)}

\author{
por \\ FERNANDO AMORES CARREDANO \\ DANIEL GONZÁLEZ ACUÑA \\ PEDRO PÉREZ QUESADA
}

RESUMEN Los estudios arqueológicos en la ciudad de Sevilla gozan de una importante tradición que puede extenderse al menos durante los últimos cien años. Sin embargo, a efectos del presente estudio hemos establecido una delimitación temporal más reducida correspondiente con los últimos cincuenta años, atendiendo a los criterios de intencionalidad científica y registro completo que inaugura Collantes de Terán en la "Cuesta del Rosario" (1944). Asimismo, se ha concentrado nuestra atención en aquellas intervenciones efectuadas en el casco y arrabales históricos de la ciudad, obviando la Cartuja de Santa María de las Cuevas, la Buhayra o el Cortijo de Miraflores. En este marco de actuación nuestro objetivo es efectuar un balance de los resultados obtenidos haciendo una revisión crítica de los análisis anteriores y avanzando en nuevas líneas de trabajo.

\begin{abstract}
Archaeological studies in the city of Seville have been developed at least during the last century. However, the time delimitation of this work is more reduced, corresponding with the last fifty years, due to scientist criteria and complex registry make by Collantes de Terán in the "Cuesta del Rosario" (1944). In the same way, our attention is focused in those interventions make in the historic center and suburbs, and not in distant ones. The objective of this work is to make an assessment of results by a critic revision of the previous analysis and to advance in new research lines.
\end{abstract}

Palabras claves Arqueología urbana; SIG; Métodos de evaluación; Patrimonio Arqueológico; Sevilla.

Key words

Urban archaeology, GIS; Evaluation methods, Archaeological heritage; Seville. 


\section{BALANCES PRECEDENTES. LA VISIÓN DESDE LA ADMINISTRACIÓN (1995)}

Haciendo una somera clasificación de las intervenciones efectuadas podemos observar un antes y un después de 1984, año en el que la Delegación de Cultura se hizo cargo de la coordinación y seguimiento de las intervenciones arqueológicas. Las excavaciones que preceden a esta fecha se distinguieron por su carácter puntual y/o estar motivadas por hallazgos casuales. La intensificación de la acción administrativa posterior supuso el incremento del número de intervenciones, en parte gracias al desarrollo del marco legislativo e institucional; cabe destacar, por ejemplo, la creación de la figura del arqueólogo provincial, que en el caso de Sevilla potenció el estudio del ámbito urbano. Se inicia por entonces un proceso de integración de la planificación patrimonial en los planes de ordenación urbana de una manera más sistemática, algo que no fue ajeno al desarrollo normativo nacional y autonómico.

Diez años después, en 1995, la Gerencia Municipal de Urbanismo efectuó un primer análisis de resultados de la Arqueología Urbana en el Conjunto Histórico de Sevilla con vistas al diseño del Plan Especial de Protección del mismo. Este primer balance supuso un avance importante dentro de la actividad arqueológica de la ciudad ya que llevaba aparejada la inclusión de la disciplina dentro de un documento de planificación urbana.

La visión institucional ${ }^{1}$ resultante de estos trabajos debía servir de reflexión previa a la elaboración de unas directrices generales de protección que se concretarían en la confección de una "carta arqueológica" (documento en el que la ciudad se encontraría zonificada en áreas homogéneas de interés arqueológico).

El análisis descriptivo resumía en número y distribución espacial las intervenciones realizadas desde 1944 hasta 1990. Se exponía una representación espacial de las intervenciones sobre el parcelario urbano (fig.1) de la cual los autores extraían una serie de conclusiones. Por un lado, se afirmaba que la acumulación y dispersión de las intervenciones por el Conjunto Histórico daba como resultado un buen conocimiento general de la ciudad. De la misma manera, se comentaba que la concentración de éstas en determinadas áreas implicaba un mayor conocimiento de dichas áreas (idea ejemplificada en la zona del antiguo núcleo poblacional).

Otro aspecto del análisis era una gráfica (fig. 2) que reflejaba el número de intervenciones por año. Esta gráfica tiene su importancia a la hora de establecer una evolución diacrónica de la actividad arqueológica de la ciudad. La lectura realizada por los autores del balance afirmaba que hasta 1989 se había producido un aumento constante de las intervenciones, constatándose desde entonces un brusco descenso en su número y llevándose a cabo tan sólo tareas de apoyo a la restauración. La explicación dada a tal evolución era que el número de las intervenciones había permitido un mayor conocimiento de la ciudad, lo que justificaba el levantamiento de las cautelas sobre ciertas zonas de protección arqueológica (Ramírez y Vargas 1996: 18). Asimismo, las intervenciones de apoyo a la restauración se debían a las actuaciones sobre edificios históricos destinados a albergar sedes institucionales.

1. Incluida en dos monografías editadas al efecto (El Conjunto Histórico de Sevilla 1995) y (Ramírez y Vargas 1996). 


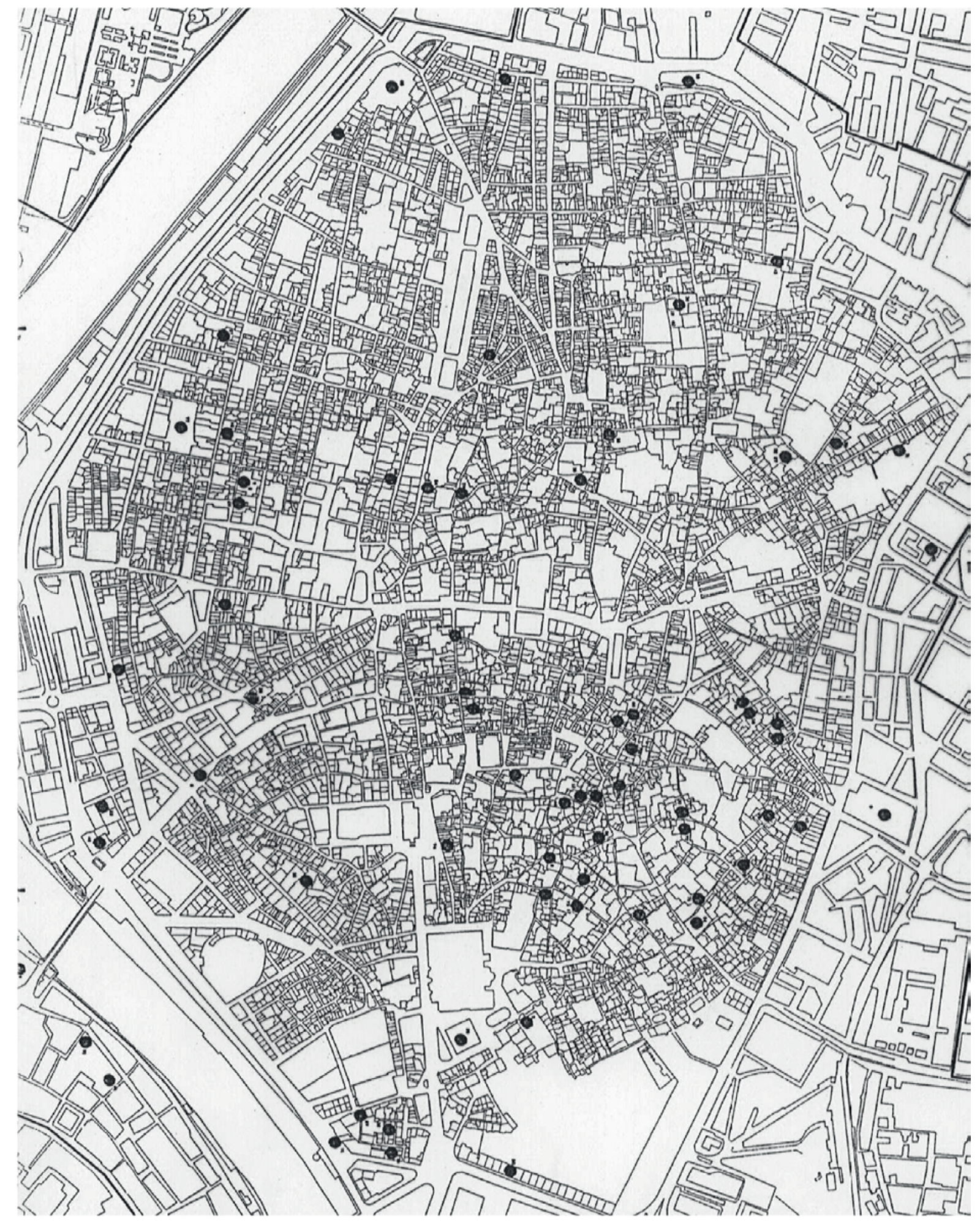

Figura 1. Localización de intervenciones arqueológicas (1944-1990) sobre el parcelario del Conjunto Histórico de Sevilla (El Conjunto Histórico de Sevilla 1995: 115, Plano II-5)

ISSN: 1133-4525 ISSN-e: 2255-3924

SPAL $9(2000)$

http://dx.doi.org/10.12795/spal.2000.i9.27 


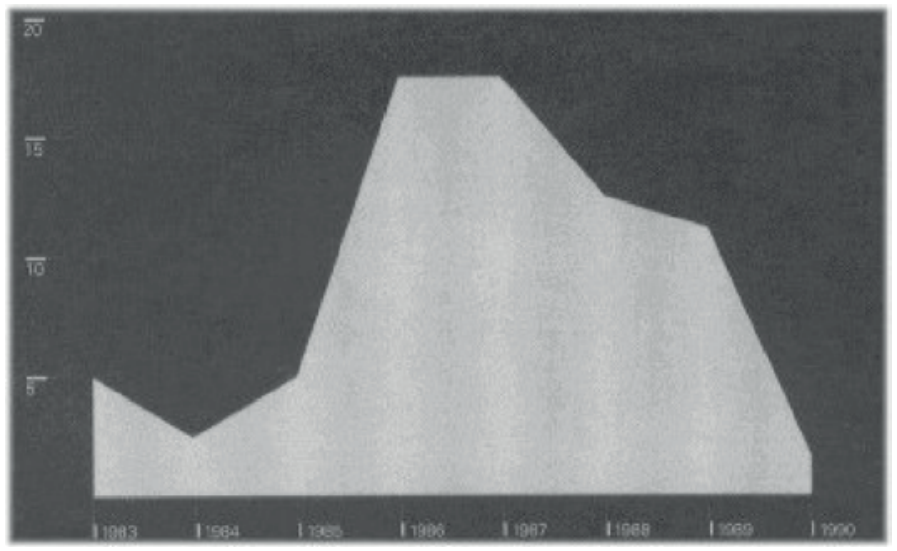

Figura 2. Diagrama de intervenciones arqueológicas anuales (1983 - 1990) (Ramírez y Vargas 1996: 17).

\section{Actualización del balance de 1995}

Como primer paso de nuestro análisis, hemos actualizado el balance de 1995 siguiendo sus mismas directrices, añadiendo las intervenciones realizadas hasta mediados de 1998, generando nuevos planos y gráficas. En el plano de localización de intervenciones (fig.3) vemos cómo se ha producido un aumento en el número de intervenciones (más del doble de las aparecidas en el balance de 1995) ${ }^{2}$. Se observa una dispersión hacia zonas periféricas, lo que podría asumirse como un mayor conocimiento global de la ciudad.

De la misma manera, la gráfica acumulativa (fig.4) refleja una revitalización de la actividad arqueológica después de 1995, superando incluso al número existente hasta 1990; lo cual pone en entredicho los argumentos ofrecidos para el relajamiento de cautelas en los años anteriores y posteriores a la Expo'92. En cualquier caso, siguiendo las premisas interpretativas del Documento de Avance de 1995 podría afirmarse un aumento del conocimiento histórico-arqueológico global de la ciudad de Sevilla.

Esta visión, con sus correspondientes conclusiones, es la plasmación patente de una serie de criterios asumidos desde la administración, que a nuestra forma de ver se resumen en los siguientes:

- Se identifica la cantidad y dispersión de las intervenciones con cantidad e incremento del conocimiento real de la ciudad.

- La concentración de intervenciones sobre un área supone un conocimiento aceptable y permite una liberación o relajamiento de cautelas arqueológicas.

- Toda intervención es un acto de investigación, cuyo resultado es conocimiento histórico.

En nuestro trabajo hemos sometido a crítica esta concepción de la realidad arqueológica urbana a la luz de nuevos datos y desde nuevos planteamientos, obteniendo los resultados que pasamos a exponer.

2. La Casa de la Moneda ha sufrido un tratamiento especial en el recuento de las intervenciones a las que ha estado sometida. Tan sólo se han llevado a cabo tres intervenciones en el citado recinto, pero debido a la dispersión interna de las actuaciones (en distintas parcelas), el solapamiento de las mismas y las necesidades de la metodología de zonificación se decidió colocar un punto en cada parcela afectada. 


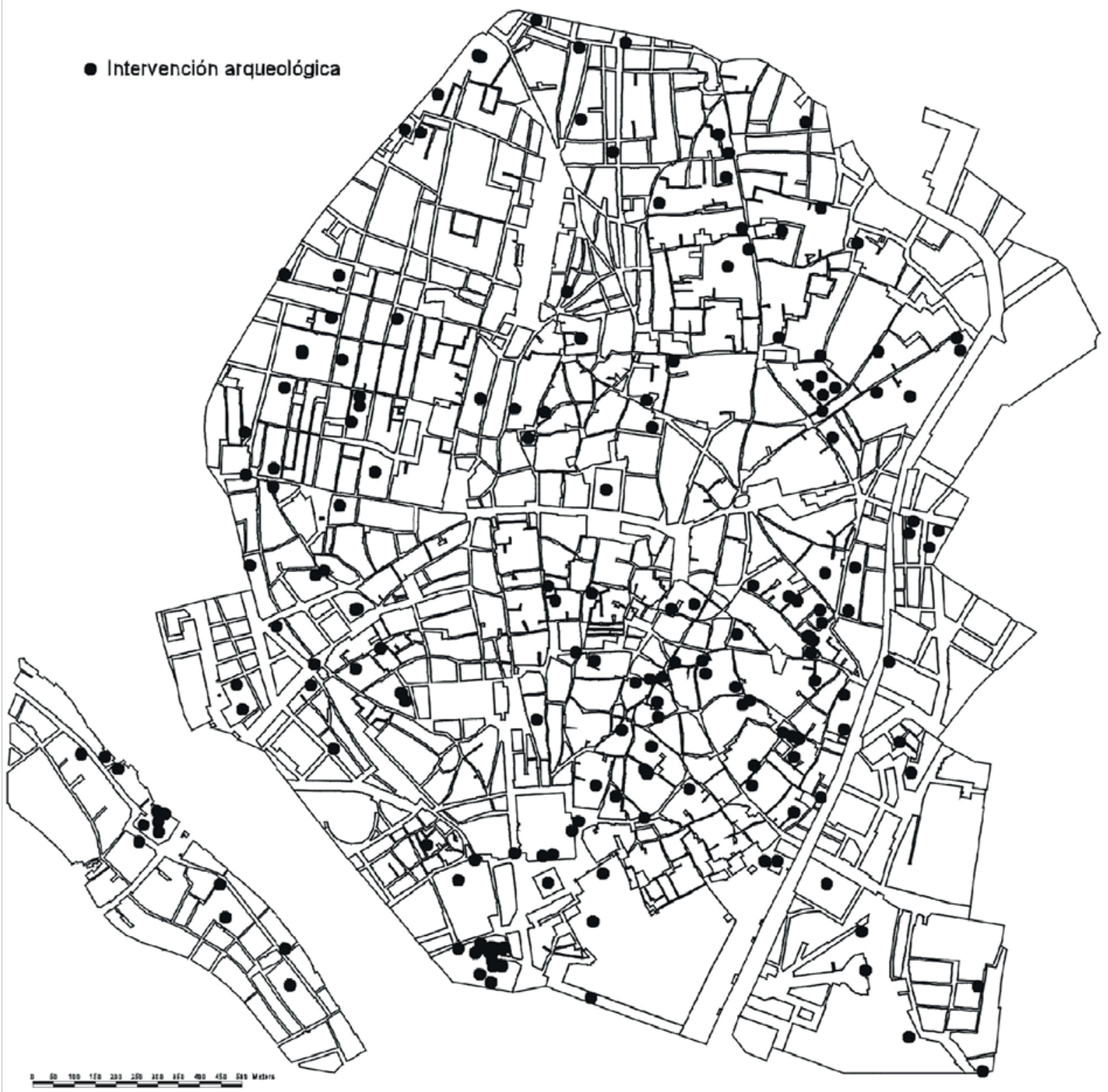

Fig. 3. Localización de intervenciones arqueológicas (1944-1998) sobre las manzanas catastrales del Conjunto Histórico de Sevilla. 


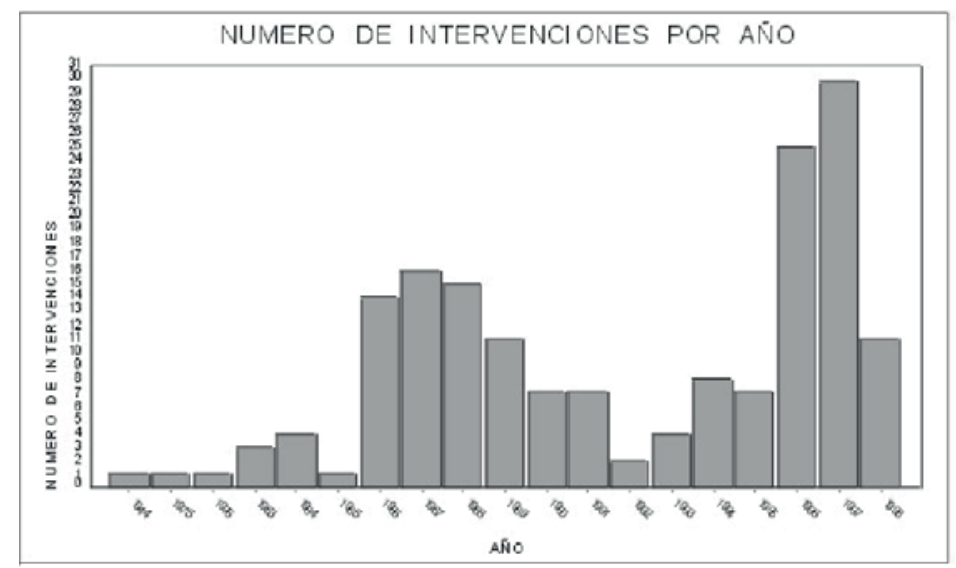

Fig. 4. Diagrama de intervenciones arqueológicas anuales (1944-1998).

\section{BALANCE CUALITATIVO DE LA ARQUEOLOGÍA URBANA EN SEVILLA (1999)}

\section{Premisas conceptuales}

En el proceso iniciado a mediados de los ochenta, de integración de la planificación patrimonial en los planes de ordenación urbana, se pueden observar dos aspectos destacados que interesan especialmente para la realización de un balance que supere al tradicional: por una parte, la consolidación de la figura de intervención de urgencia, y por otra, el reconocimiento de la importancia de la zonificación urbana para la planificación y toma de decisiones.

Sobre el primero de estos aspectos cabe aclarar una situación jurídica poco precisa. La Autorización de Actividad Arqueológica de Urgencia se contempla por el Reglamento de Actividades Arqueológicas (1993) como una de las tres modalidades de intervención sobre el Patrimonio Arqueológico Andaluz ${ }^{3}$. Se entiende la Actividad Arqueológica de Urgencia como un procedimiento simplificado para la autorización en aquellas situaciones en que concurran circunstancias de peligro de pérdida o destrucción de los bienes del Patrimonio Arqueológico Andaluz. Este tipo de procedimiento es el aplicable en áreas que cuenten con protección arqueológica, según rige el Reglamento de Protección y Fomento del Patrimonio Histórico de Andalucía (1995) y, de hecho, es el que se aplica en el Conjunto Histórico de Sevilla. El expediente administrativo se resuelve con la presentación de un Informe en el plazo de un mes desde la finalización de la actividad y de una Memoria Científica en el plazo máximo de un año.

Aun cuando entendemos que, por inercia contextual, este acto administrativo se considera como investigación, nosotros pensamos de hecho que se trata de una acción de protección de la información en aras de no entorpecer la propia dinámica urbana, en la que el Patrimonio Arqueológico no es sino un ingrediente más en la gestión y planificación de la ciudad, que sólo excepcionalmente puede asumir la conservación de estructuras arqueológicas.

3. Las otras modalidades son, de un lado, las enmarcadas en Proyectos Generales de Investigación y, de otro, las autorizaciones de Actividades Arqueológicas no incluidas en un Proyecto General de Investigación. 
La inexistencia de un proyecto arqueológico para la ciudad, que proporcione hipótesis previas e interprete resultados, incrementa la confusión entre investigación y acto administrativo. En la actualidad la confusión entre estos aspectos obliga a la improvisación de hipótesis que el arqueólogo urbano trata de contrastar en su particular parcela de intervención. Sin embargo, en nuestra opinión y desde una posición pragmática, en ausencia de un Proyecto General de Investigación, la opción más correcta es concentrar los esfuerzos en la salvaguarda de la información y de los restos materiales de la forma más adecuada posible. Lo importante de una intervención de urgencia al día de hoy (recordemos que no existe un Proyecto de Investigación sobre el yacimiento de Sevilla como tal) es posibilitar las interpretaciones futuras a partir del registro arqueológico rescatado, aún cuando entendamos la necesidad de plantear hipótesis previas y de hacer interpretaciones en el momento de intervenir ${ }^{4}$.

Lo que no creemos es que la intervención de urgencia sea de hecho sinónima de investigación y conocimiento histórico, como parecen entender los autores del Documento de Avance del Plan Especial. Entendemos que hay que diferenciar el conocimiento actual del cual disponemos a partir de interpretaciones parciales, de aquel conocimiento que se derivaría de la intensa investigación de los contextos parciales recuperados situándolos en el marco del yacimiento ciudad de Sevilla.

Habría que replantear, por tanto, los criterios apuntados en 1995 por la Gerencia de Urbanismo:

- Desde un punto de vista cuantitativo y temporal, planteamos que frente a la identificación de una proporcionalidad estricta entre la cantidad de intervenciones y la cantidad del conocimiento acumulado hay que considerar que el resultado de las intervenciones no es conocimiento sino información; por tanto, el valor acumulado no depende exclusivamente del número de intervenciones sino que está determinado en primera instancia por la calidad del registro de esa información; es decir, depende de la capacidad que el registro administrativo resultante proporciona para su interpretación futura. Ese potencial informativo es el que nosotros hemos entendido como clave para realizar un balance de las intervenciones arqueológicas urbanas (Amores y otros 1999).

- Desde un punto de vista espacial, no compartimos la identificación que se hace de distribución uniforme de intervenciones con incremento del conocimiento general de la ciudad; y consecuentemente tampoco compartimos que una concentración de intervenciones sobre un área de la ciudad suponga un conocimiento aceptable de la misma y una justificación para la relajación de sus cautelas arqueológicas. Por contra, pensamos que la localización puntual de las intervenciones, aunque es un importante instrumento para la gestión de la información patrimonial, no ofrece una visión correcta del potencial informativo para el conjunto urbano. Como ya señalaba el Documento de Avance del Plan Especial, ha de producirse un análisis exhaustivo de la documentación [.... ] que conduzca a establecer zonificaciones homogéneas previas, jerarquías de interés y evaluación del "riesgo arqueológico" (El Conjunto Histórico de Sevilla 1995: 80). En este sentido, nosotros creemos que para establecer un balance espacial del fenómeno de las intervenciones arqueológicas urbanas resulta adecuada una zonificación del potencial informativo acumulado.

A partir de este planteamiento teórico podemos señalar los dos objetivos fundamentales de nuestro trabajo, cada uno de los cuales tiene un desarrollo metodológico diferente:

1. Evaluar las intervenciones urbanas realizadas, mediante el análisis del potencial informativo de su asiento administrativo.

2. Deducir a partir de los valores puntuales de cada intervención una zonificación de la ciudad en grados de potencial informativo, de acuerdo con el posible aporte de información entre intervenciones próximas.

4. Somos conscientes de que la Arqueología es una disciplina intencional y de que los datos por sí solos, por muy bien que estén recogidos, no hablan. De ahí la importancia de las actuaciones de investigación completas y de las revisiones de estos datos. 


\section{Metodología de evaluación de intervenciones}

Para obtener una secuencia ordenada de las intervenciones de acuerdo con su potencial informativo hemos empleado técnicas y conceptos pensados especialmente para el mundo empresarial de cara a la toma de decisiones en función de múltiples criterios, y que hemos adaptado en forma de método de evaluación multicriterio y multiatributo.

Entre los conceptos teóricos más importantes que hemos asumido están:

a) El concepto de punto ideal, que utilizan algunos teóricos de la economía para definir aquella alternativa que mejor cubre las especificaciones deseadas y que el decisor elegiría sin dudar si pudiera. En nuestro caso de estudio pensamos que la mejor alternativa sería aquella intervención que mejor y más aporte de información tiene documentado en su asiento administrativo o, en su defecto, en los anuarios arqueológicos y publicaciones monográficas.

b) La división del problema en varios niveles jerárquicos que definen la naturaleza de las alternativas (la idea original la hemos tomado del Método de Jerarquías Analíticas -Analytic Hierarchy Process- de Saaty (1977, 1980 y 1988). En concreto nosotros hemos establecido tres niveles jerárquicos que sean capaces de abarcar los aspectos más importantes de cualquier intervención: niveles de criterios, atributos y categorías:

- Nivel de CRITERIOS: cada punto de vista desde el cual se procede a la valoración de la realidad analizada.

Entendemos que se pueden diferenciar tres grandes criterios o tipos de intervención, atendiendo a la metodología seguida en el proceso de registro arqueológico:

- Vigilancia o seguimiento de obra: es el tipo de intervención más elemental y en realidad no es una excavación arqueológica en sentido estricto, sino un control de obra. En estas intervenciones el mismo proceso de recogida de información limita enormemente la calidad del registro y de las interpretaciones.

- Registro mediante niveles arbitrarios: se puede considerar un paradigma de excavación arqueológica, hoy día en desuso, cuya característica más sobresaliente es el procedimiento de registro mediante la profundización estableciendo niveles arbitrarios o artificiales.

- Registro de las unidades estratigráficas: es el paradigma de excavación actualmente establecido; se considera el más adecuado para el registro ya que es el que mejor garantiza la contextualización de la información extraída.

- Nivel de ATRIBUTOS: cada una de las propiedades objetivas que puede presentar el registro administrativo como resultado de la intervención y que la caracterizan, por ejemplo planimetría, documentación gráfica, analítica...

- Nivel de CATEGORÍAS: cada una de las opciones que pueden concretar la presencia de un atributo, por ejemplo, dentro del atributo documentación gráfica hemos considerado tres categorías, ausente, general y de detalle.

Mediante la ponderación de criterios, atributos y categorías se facilita la valoración de cada intervención según sea la combinación presente en cada registro. La gama de combinaciones que se obtiene por este procedimiento es enorme y después de haber examinado los informes y memorias presentes en la Delegación Provincial de Cultura podemos decir que la ficha utilizada abarca suficientemente la diversidad de presentaciones que ofrecen estos documentos (fig. 5). 


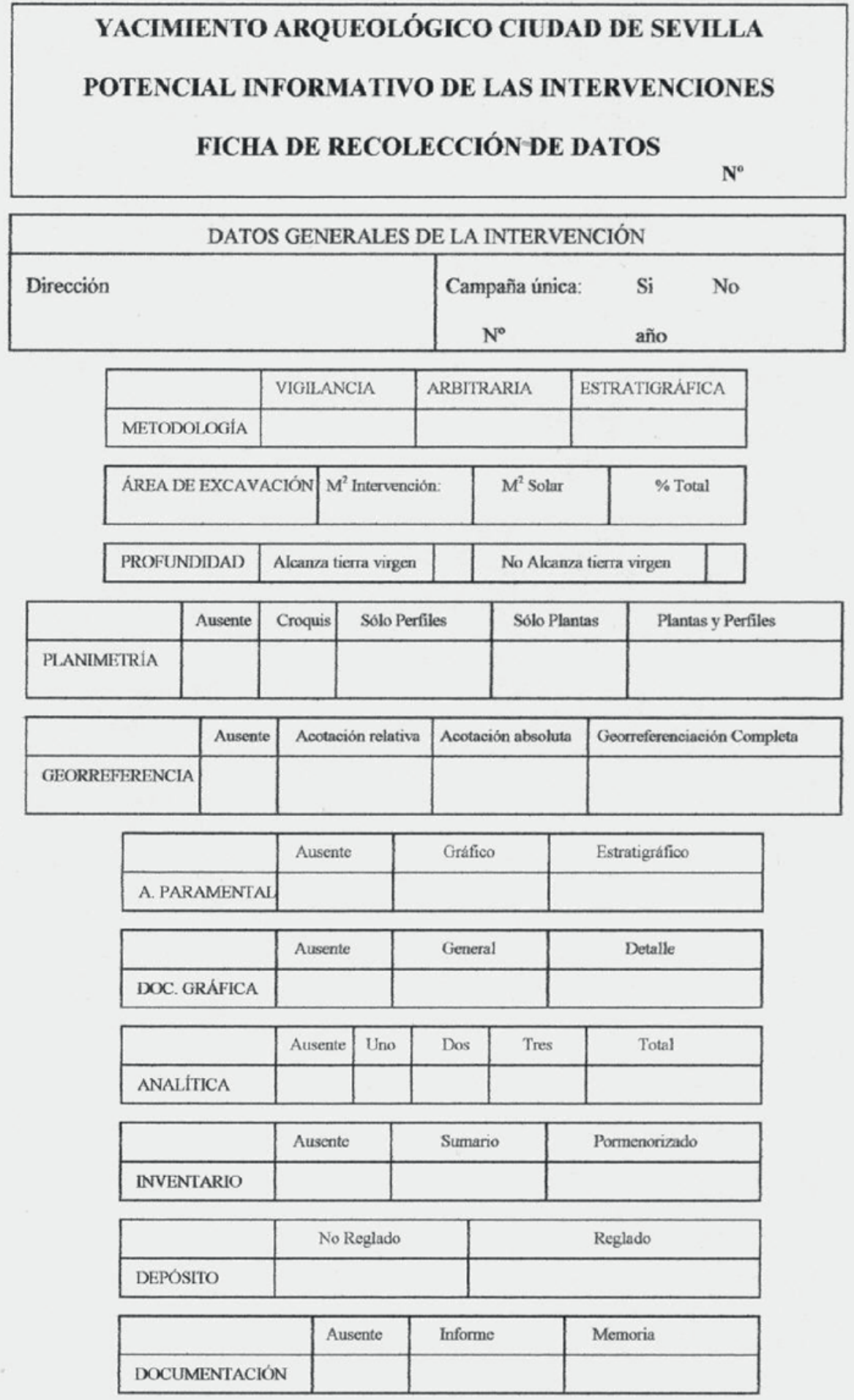

Figura 5. Ficha de recolección de datos. 
El resultado de constatar las características detectadas en cada informe, memoria o licencia de obra ha sido un valor individual para cada intervención. Los resultados se han normalizado en un rango de valores entre cero y mil, de manera que el valor máximo (mil) indica el punto ideal mientras que el mínimo (cero) señala el anti-ideal (Fig. 6).

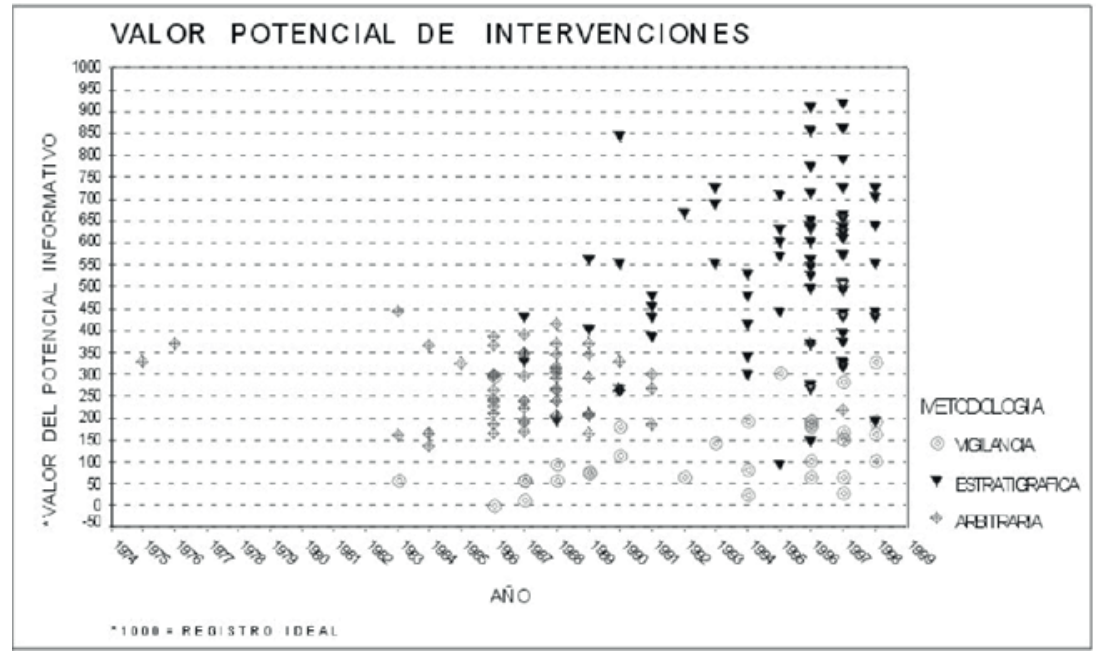

Figura 6. Dispersión de valores de intervención (Gráfica de nube de puntos). Esta gráfica expresa visualmente el valor obtenido por cada intervención que la sitúa entre el ideal y el anti-ideal, de manera que su distancia a estos extremos define su potencial informativo en función de las ponderaciones realizadas y en relación con el resto de intervenciones.

A partir de la evaluación y ordenación de las intervenciones arqueológicas ya podemos presentar un balance para la ciudad de Sevilla, centrándonos en su análisis temporal y espacial.

Sobre la metodología de evaluación cabe aclarar una idea importante: lo que se evalúa no es la eficiencia (es decir, la consecución de los objetivos de la actividad de urgencia con el mínimo gasto de energía, tiempo, dinero o esfuerzo) mostrada por los arqueólogos urbanos en sus actuaciones individuales. Ya se sabe que las disponibilidades presupuestarias son diversas en las intervenciones arqueológicas urbanas, y por tanto no podemos evaluar la eficiencia si no contamos con el conocimiento de estos presupuestos y otros aspectos coyunturales aún más imprecisos. Por tanto, lo que se evalúa no es la eficiencia del excavador sino la eficacia de la intervención, es decir la calidad de los resultados obtenidos, y sin valorar la calidad de las interpretaciones historiográficas.

\section{Resultados del análisis temporal}

Una primera aproximación al fenómeno de urgencias desde el punto de vista cuantitativo y temporal lo podemos observar en la figura 7 , donde se muestra un resumen anual de intervenciones discerniendo entre las distintas metodologías de intervención que hemos considerado (vigilancias, arbitrarias y estratigráficas), a través de las cuales podemos intuir una diferencia en la calidad y cantidad de información proporcionada por cada una de ellas. El diagrama supone ya una nueva visión del fenómeno frente al recuento indiscriminado que se ofrecía por la Gerencia de Urbanismo. 


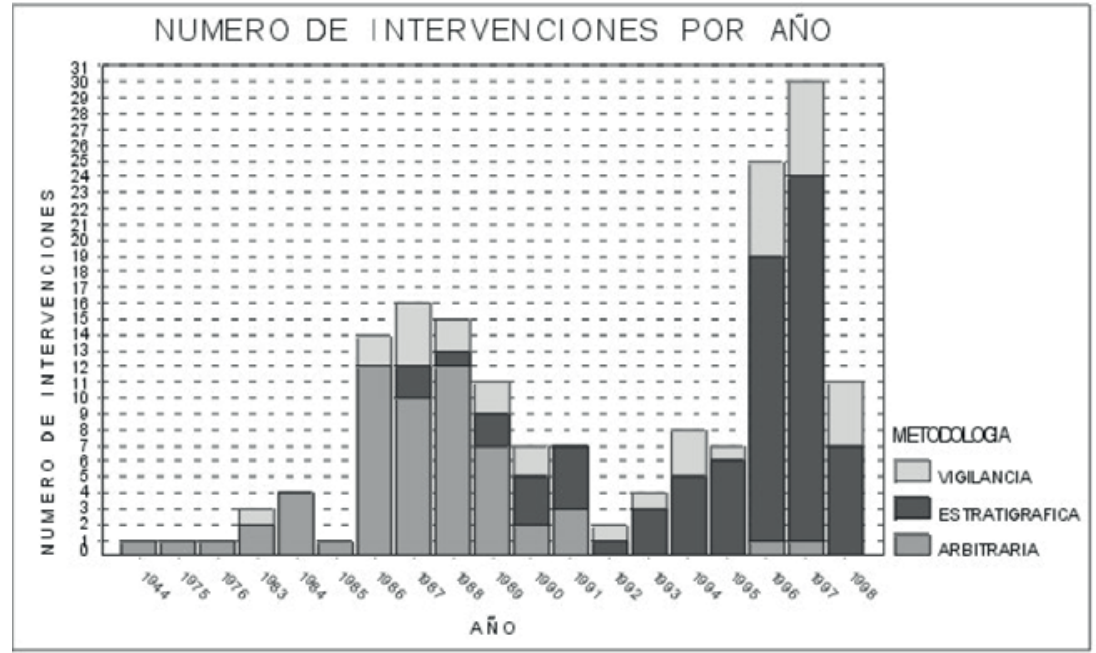

Figura 7. Diagrama de tipologías de intervención.

Sin embargo, la diferente calidad (potencial) de la información no aparece reflejada anualmente. La evolución del potencial informativo de los informes y memorias está representada en el siguiente diagrama (Fig. 8), en el que se expresan los valores obtenidos acumulados en cada año.

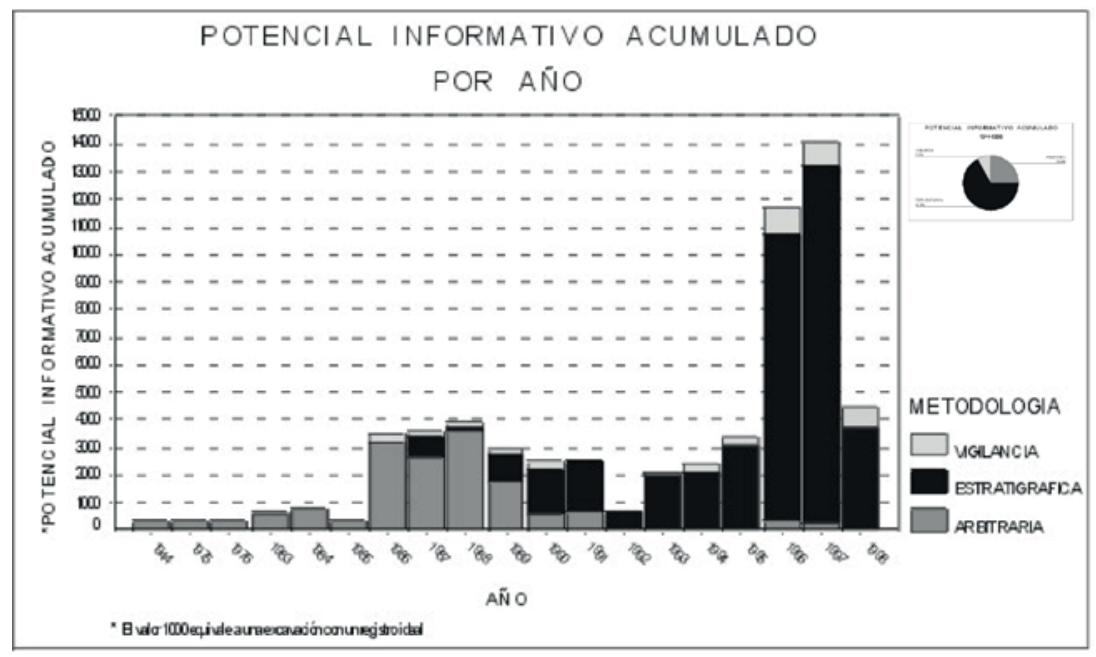

Figura 8. Diagrama de valores potenciales anuales

De la comparación de estas gráficas se pueden hacer algunas lecturas. Por ejemplo, hay años muy significativos como el 87 y el 94 en los que realizan más intervenciones que los años inmediatamente anteriores y posteriores a cada uno y, sin embargo, la información acumulada los equilibra bastante con estos años. Si observamos el diagrama de tipologías (fig.7) podemos entender a qué se debe este fenómeno: son años en los que realizan muchas vigilancias para cubrir el expediente administrativo y la calidad en el registro de la información se muestra ciertamente insignificante. 
Lo más destacado que podemos concluir de estos diagramas es la evolución de la arqueología urbana en la ciudad de Sevilla, tanto cuantitativa como cualitativamente. En primer lugar, en el corto período de tiempo que va desde mediados de los ochenta hasta finales de los noventa se pueden observar fácilmente dos períodos álgidos y uno de recesión en la actividad arqueológica. Esta recesión, como ya se dijo, se justificaba en el Documento de Avance del Plan Especial por el mejor conocimiento de la ciudad. Sin embargo, nuestra opinión al respecto difiere sustancialmente y encuentra justificada esta disminución de intervenciones en unos ritmos y circunstancias socioeconómicas que terminan afectando a la actividad arqueológica. Cabe citar, por ejemplo, la renovación de normativas jurídicas (Patrimonio Histórico y Suelo), que influyen en la adopción de medidas más o menos ejecutables pero siempre influyentes; el planeamiento urbanístico y la variabilidad en la dinámica edificatoria, en función de una escala macroeconómica que influye en la intensidad de la construcción (por ejemplo, Expo '92); y las alternancias políticas y administrativas a todos los niveles, expresadas en cambios sucesivos de criterios en el seno de la Comisión Provincial de Patrimonio Histórico ${ }^{5}$, aparecen a priori como argumentaciones más razonables que las esgrimidas en el balance de 1995.

Por lo que se refiere a la evolución cualitativa de las intervenciones los aspectos implicados son de diferente naturaleza si bien pueden estar mediatizados por las circunstancias administrativas y de mercado, como así ocurre, por ejemplo, con el difícil desarrollo profesional de la arqueología urbana. Lo más destacado e influyente en el sentido cualitativo será, no obstante, la propia tradición y cambio que se produce en la metodología arqueológica y en la reflexión patrimonial (Fig. 9).

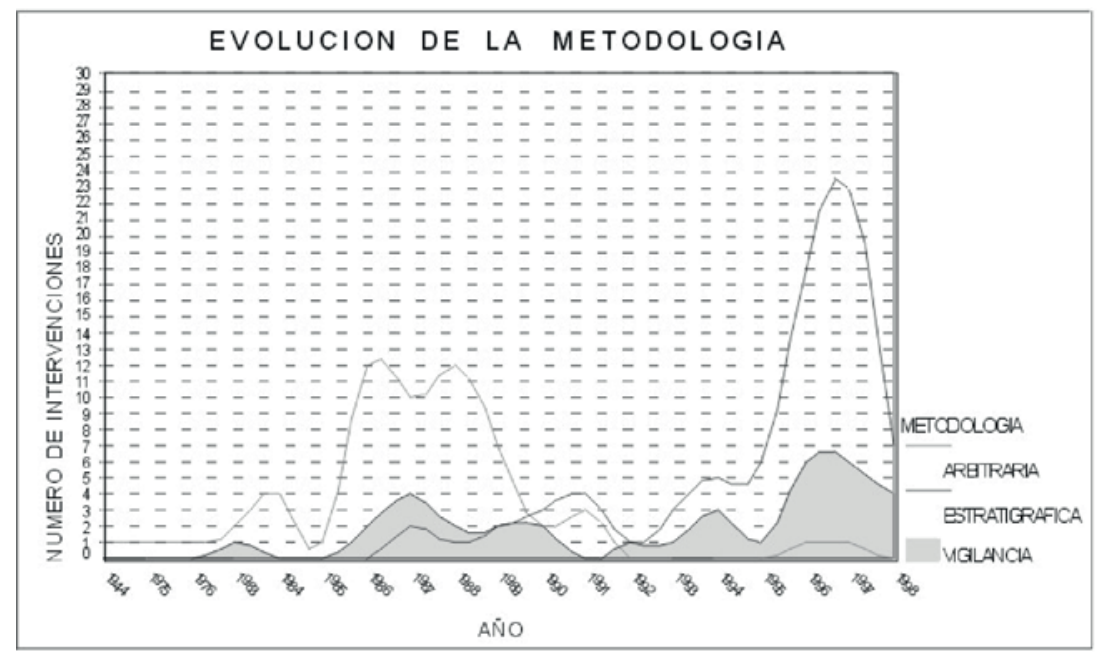

Figura 9. Evolución cuantitativa de las metodologías de intervención.

Se puede observar claramente cómo en los años de "crisis" se produce un cambio de paradigma referido al tipo de intervención: a finales de los ochenta comienza a sustituirse el paradigma hasta entonces imperante, la excavación con metodología arbitraria, por el actual paradigma de excavación, con metodología estratigráfica. Por su parte las vigilancias de obras se han consolidado como una forma de intervención importante en todo el período contemplado, habiendo sobrevivido al cambio de paradigma y mostrándose de gran importancia numérica (suponen aproximadamente la quinta parte de las intervenciones) manteniendo anualmente unos porcentajes constantes en relación con los otros tipos de intervención.

5. La consulta de los Anuarios Arqueológicos de Andalucía (1985-1993), revela claramente esta diferencia de criterios en Sevilla donde se han sucedido cada 2/3 años los responsables provinciales de arqueología. 
El cambio de paradigma será responsable en gran medida de una mejora cualitativa en el registro de la información como se puede observar en la gráfica acumulativa del potencial informativo de las intervenciones (Fig. 10).

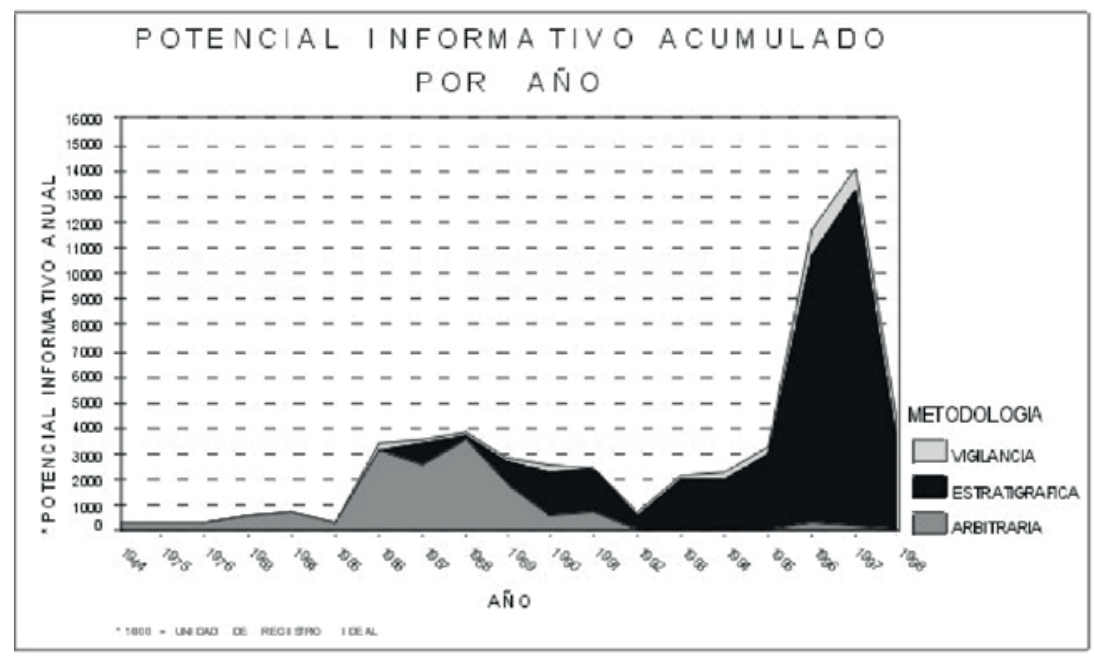

Figura 10. Evolución cualitativa de las metodologías de intervención.

El potencial informativo acumulado por las intervenciones estratigráficas se muestra sensiblemente superior al de las arbitrarias; se puede decir que se ha mejorado en calidad de registro. Por su parte, las arbitrarias y especialmente las vigilancias contrastan su potencial informativo con su expectativa numérica, siendo su aportación ínfima en el caso de las vigilancias.

No obstante esta última lectura tiene sus matices si recordamos la gráfica de dispersión de valores de intervención de la figura 6 . En ella se observa cómo han ido mejorado sensiblemente en calidad las vigilancias aún cuando todavía hoy no alcanzan niveles aceptables (por encima del punto medio $=500$ ). Las intervenciones arbitrarias, cuando estaban en uso, tenían un rango estrecho de valores medios o bajos no alcanzando en ningún caso un nivel aceptable. De hecho, en el caso de las arbitrarias hemos podido observar que este auge resultó ciertamente perverso, estandarizándose la documentación hasta tal punto que se copiaban literalmente los informes previos para la solicitud de autorización. Sobre las intervenciones estratigráficas se puede observar una amplia variedad de situaciones que en términos generales presenta un incremento paulatino de los valores medios de las intervenciones. No obstante, el rango de valores es muy amplio y mientras en algunos casos se presentan informes con metodología estratigráfica ciertamente pobres, en otros se alcanzan valores próximos al ideal, como en Castilla 51 y en la Acera de Levante de la Catedral. Destaca también el Real Monasterio de San Clemente que ya en 1990 alcanza un valor realmente sobresaliente.

\section{Zonificación y análisis espacial}

Una vez que hemos obtenido unos valores individuales y hemos analizado la situación de las actividades arqueológicas urbanas en el tiempo, una primera aproximación a la representación del potencial informativo en el parcelario urbano puede ser la gradación en colores y su distribución puntual. De esta manera, si establecemos a partir de la gráfica anterior tres intervalos de valor (bajo, medio y alto) la visión del fenómeno tiene la siguiente distribución (Fig. 11): 


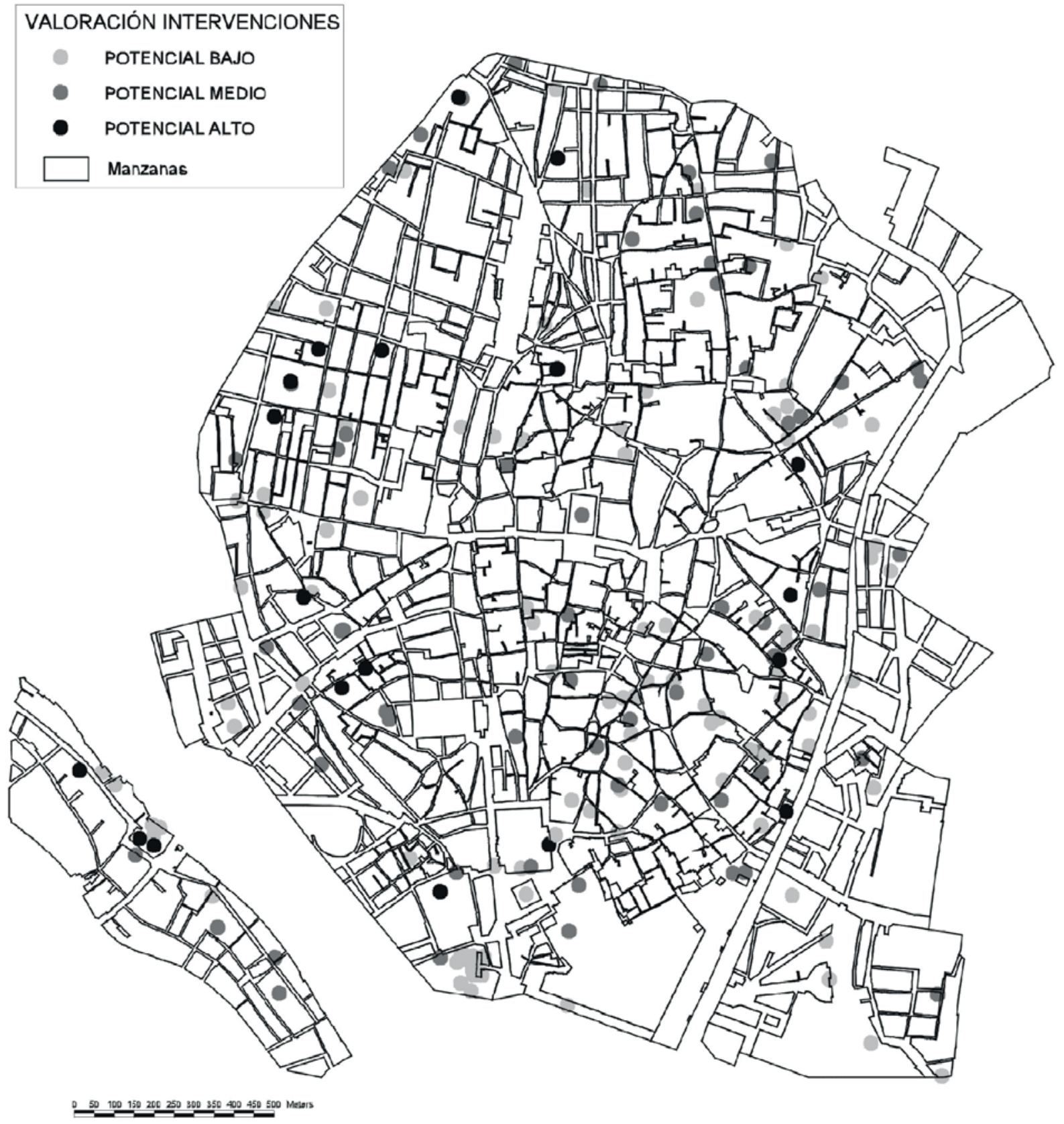

Fig. 11. Distribución espacial de intervenciones según intervalos de valor.

De esta distribución podemos apuntar algunas ideas: se comprueba un alto número de intervenciones con potencial bajo y distribución uniforme. Además, se observa una concentración importante en el sector SE, el más antiguo de la ciudad. Existe un número aceptable de intervenciones con potencial medio y distribución uniforme. El número de intervenciones con potencial alto es escaso, distribuyéndose en áreas periféricas de la ciudad histórica. 


\section{Metodología de zonificación de la ciudad}

Una vez llegados a este punto, debemos recordar cual era el segundo objetivo fundamental de nuestro trabajo: deducir a partir de los valores puntuales de cada intervención una zonificación de la ciudad en grados de potencial informativo, de acuerdo con el posible aporte de información entre intervenciones próximas. Ahora bien ¿cómo zonificar la ciudad a partir de valoraciones puntuales?

La solución a este problema la hemos facilitado con el establecimiento de unos pasos metodológicos, partiendo de la siguiente premisa: el potencial de información en la ciudad se enriquece por la adición de valores de las intervenciones cercanas. Los pasos metodológicos han sido:

\section{Extrapolación}

$1^{\circ}$. Extrapolación de valores puntuales en un área de influencia que se ha establecido en 100 metros de radio. La elección de esta distancia es sólo una aproximación que hemos elegido como abstracción de una realidad, que es lo que en definitiva supone la zonificación de cualquier fenómeno. Hemos entendido que la interrelación de la información de intervenciones próximas se puede hacer con cierto grado de verosimilitud hasta esta distancia ${ }^{6}$.

$2^{\circ}$. Adición de valores en las zonas de contacto. Las áreas de contacto entre intervenciones próximas adquieren mayor potencial interpretativo al disponer de una mayor diversidad en las fuentes de información.

$3^{\circ}$. Reclasificación de los valores obtenidos en cinco intervalos (muy alto, alto, medio...) para obtener una primera zonificación del fenómeno.

El resultado ha sido una primera aproximación a la zonificación pretendida (Fig. 12), en la que ya podemos observar unas tendencias zonales, pero que son poco claras de cara al establecimiento de zonas homogéneas para la planificación urbana.

Para corregir la difícil lectura que se puede hacer del fenómeno a partir de este mapa, hemos realizado un segundo paso metodológico:

\section{Interpolación}

$1^{\circ}$. A partir de los valores obtenidos en las zonas de contacto se han realizado unos procesos de interpolación. La idea es establecer una modelización del terreno similar a la empleada en los cálculos de curvas de nivel a partir de puntos muestrales, para lo cual se ha empleado uno de los métodos de ponderación por medias móviles, el denominado Kriging.

$2^{\circ}$. Una última reclasificación de los valores obtenidos en cinco intervalos facilita finalmente la comprensión de este complejo fenómeno espacial (Fig. 13).

A partir de esta distribución del potencial informativo creemos que aportamos una visión sustancialmente diferente a la del balance de la Gerencia de Urbanismo de 1995.

6. Actualmente, estamos desarrollando estos modelos de extrapolación aplicando un sistema de distancias concéntricas de diverso radio, a partir de cada intervención, que pueden matizar este factor de extrapolación. 


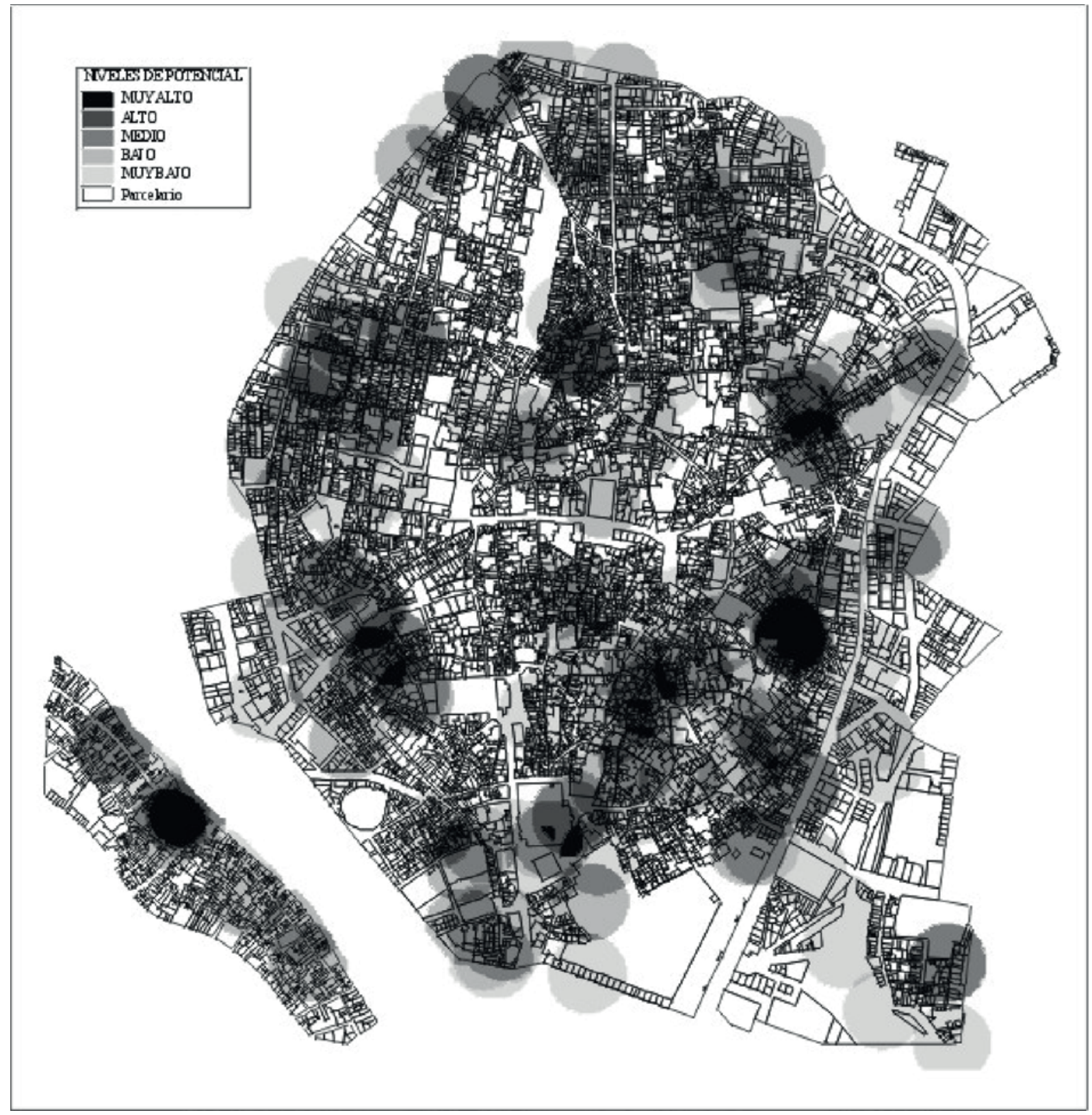

Fig. 12. Extrapolación de los niveles de potencial informativo de las intervenciones. 


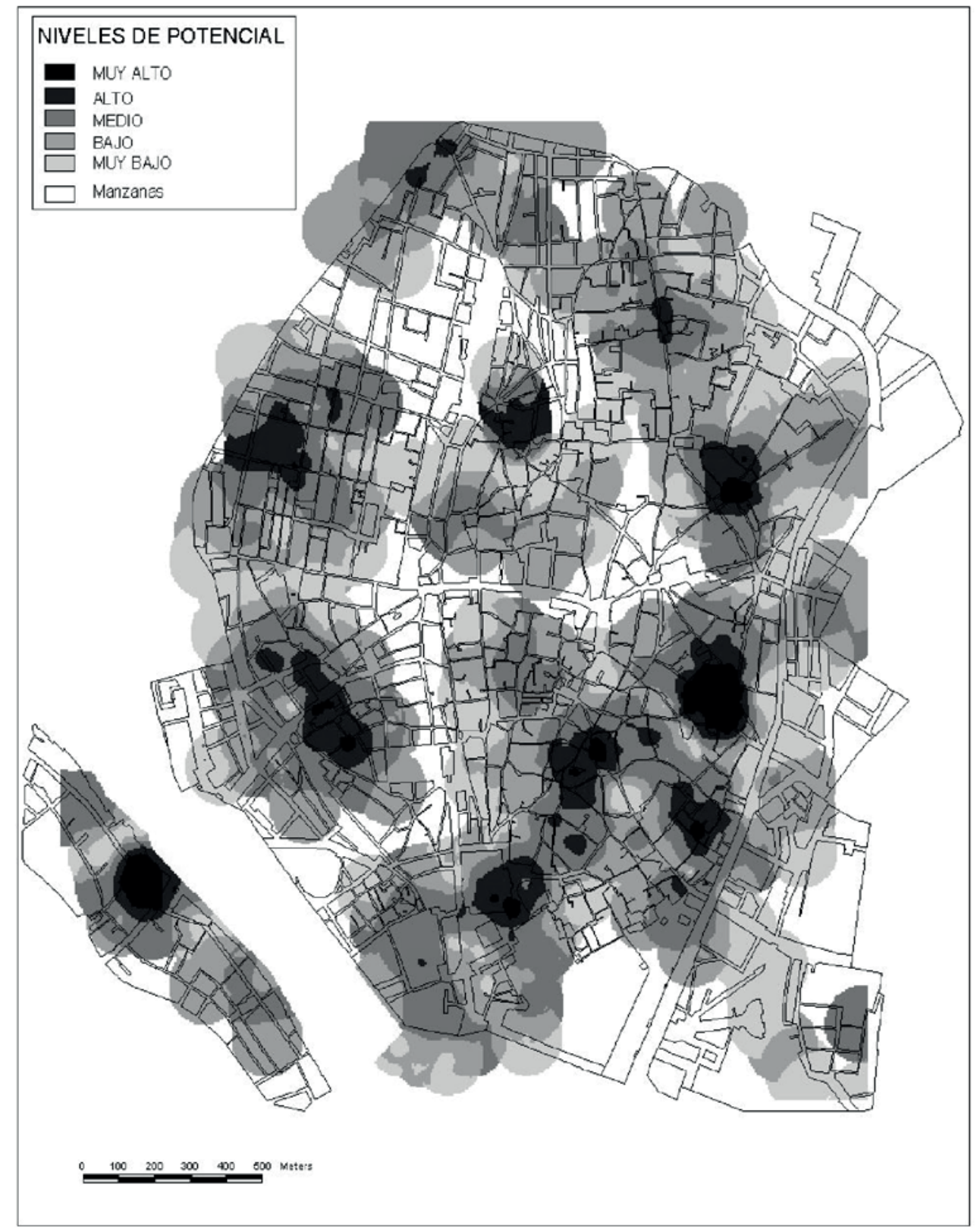

Fig. 12. Extrapolación de los niveles de potencial informativo de las intervenciones. 
Sobre los resultados del estudio del potencial informativo de las actividades arqueológicas en Sevilla se pueden hacer unas primeras lecturas:

- Tan sólo pequeñas zonas de la ciudad alcanzan niveles sobresalientes para su interpretación futura: aquellas con niveles alto o muy alto, y que muestran una distribución periférica en la mitad Sur y especialmente en el Sudeste de la ciudad. Estos niveles altos en la zona Sudeste tienen su explicación en la enorme cantidad de intervenciones de niveles bajo y medio, que por adición terminan proporcionando esos niveles altos. Además, la distribución de este alto potencial no es uniforme en la zona.

- En contraposición, las zonas más densas de potencial informativo muy alto, que podemos localizar en la zona Este y en San Jorge, se deben a la cercanía de unas pocas intervenciones de alto potencial.

- El conjunto de la ciudad muestra una gran proporción de valores nulos, muy bajos o bajos, a partir de los cuales la realización de investigaciones contaría con documentación arqueológica escasa o de muy baja calidad.

Como instrumento de tutela los resultados obtenidos en el proceso de evaluación y zonificación tienen unas ventajas importantes, derivadas especialmente de estar documentadas como bases de datos válidas para la elaboración de estrategias y toma de decisiones administrativas. En este sentido hay que decir que la tecnología utilizada posibilita el cruce de información con otras bases de datos urbanas georreferenciadas (estado de conservación del yacimiento, riesgos diversos, distribución del registro arqueológico remanente...) facilitando, en definitiva, conclusiones diferentes en función de objetivos de decisión o evaluación distintos a los aquí presentados; en relación con lo cual hay que señalar que el trabajo de evaluación no es finalista y requiere actualizaciones.

\section{BIBLIOGRAFÍA}

AMORES, F.; GONZÁLEZ ACUÑA, D.; PAVÓN, I. y PÉREZ QUESADA, P. (1999): “La evaluación del potencial informativo de las intervenciones arqueológicas en los yacimientos urbanos: aplicación del modelo para la ciudad de Sevilla”, Actas del XXV Congreso Nacional de Arqueología. Valencia. Diputación de Valencia.

EL CONJUNTO HISTÓRICO DE SEVILLA (1995): El Conjunto Histórico de Sevilla. Avance del Plan Especial de Protección. Sevilla. Ayuntamiento. Gerencia de Urbanismo.

RAMÍREZ, F. O. y VARGAS, J. M. (1996): Arqueología Urbana en Sevilla (1944-1990). Sevilla. Ayuntamiento. Gerencia de Urbanismo.

REGLAMENTO DE ACTIVIDADES ARQUEOLÓGICAS (1993): Decreto 32/1993, de 16 de marzo, por el que se aprueba el Reglamento de Actividades Arqueológicas. BOJA no 46, de 4 de mayo.

REGLAMENTO DE PROTECCIÓN Y FOMENTO DEL PATRIMONIO HISTÓRICO DE ANDALUCÍA (1995): Decreto 19/1995, de 7 de febrero, por el que se aprueba el Reglamento de Protección y Fomento del Patrimonio Histórico de Andalucía. BOJA nº 43, de 17 de marzo.

SAATY, T. L. (1977): “An Scaling Method for Priorities in Hierarchical Structures”, Journal of Mathematical Psychology: 234-281.

— (1980): The Analityc Hierarchy Process. Nueva York. Mc Graw Hill. (Versión ampliada en 1988). 